\title{
Leveraging routine clinical materials and mobile technology to assess CBT fidelity: the Innovative Methods to Assess Psychotherapy Practices (imAPP) study
}

Shannon Wiltsey Stirman ${ }^{1 *}$ D, Luana Marques², Torrey A. Creed ${ }^{3}$, Cassidy A. Gutner ${ }^{4}$, Robert DeRubeis 5 , Paul G. Barnett ${ }^{6}$, Eric Kuhn', Michael Suvak ${ }^{7}$, Jason Owen ${ }^{1}$, Dawne Vogt ${ }^{4}$, Booil Jo ${ }^{8}$, Sonja Schoenwald ${ }^{9}$, Clara Johnson ${ }^{10}$, Kera Mallard ${ }^{10}$, Matthew Beristianos ${ }^{10}$ and Heidi La Bash ${ }^{10}$

\begin{abstract}
Background: Identifying scalable strategies for assessing fidelity is a key challenge in implementation science. However, for psychosocial interventions, the existing, reliable ways to test treatment fidelity quality are often labor intensive, and less burdensome strategies may not reflect actual clinical practice. Cognitive behavioral therapies (CBTs) provide clinicians with a set of effective core elements to help treat a multitude of disorders, which, evidence suggests, need to be delivered with fidelity to maximize potential client impact. The current "gold standard" for rating CBTs is rating recordings of therapy sessions, which is extremely time-consuming and requires a substantial amount of initial training. Although CBTs can vary based on the target disorder, one common element employed in most CBTs is the use of worksheets to identify specific behaviors and thoughts that affect a client's ability to recover. The present study will develop and evaluate an innovative new approach to rate CBT fidelity, by developing a universal CBT scoring system based on worksheets completed in therapy sessions.
\end{abstract}

Methods: To develop a scoring system for CBT worksheets, we will compile common CBT elements from a variety of CBT worksheets for a range of psychiatric disorders and create adherence and competence measures. We will collect archival worksheets from past studies to test the scoring system and assess test-retest reliability. To evaluate whether CBT worksheet scoring accurately reflects clinician fidelity, we will recruit clinicians who are engaged in a CBT for depression, anxiety, and/or posttraumatic stress disorder. Clinicians and clients will transmit routine therapy materials produced in session (e.g., worksheets, clinical notes, session recordings) to the study team after each session. We will compare observer-rated fidelity, clinical notes, and fidelity-rated worksheets to identify the most effective and efficient method to assess clinician fidelity. Clients will also be randomly assigned to either complete the CBT worksheets on paper forms or on a mobile application (app) to learn if worksheet format influences clinician and client experience or differs in terms of reflecting fidelity.

Discussion: Scoring fidelity using CBT worksheets may allow clinics to test fidelity in a short and effective manner, enhancing continuous quality improvement in the workplace. Clinicians and clinics can use such data to improve clinician fidelity in real time, leading to improved patient outcomes.

Trial registration: ClinicalTrials.gov NCT03479398. Retrospectively registered March 20, 2018.

Keywords: Fidelity, Measurement, Behavioral health

\footnotetext{
* Correspondence: sws1@stanford.edu; Shannon.Wiltsey-Stirman@va.gov

${ }^{1}$ National Center for PTSD, VA Palo Alto HCS and Stanford University

Department of Psychiatry and Behavioral Sciences, 795 Willow Road, Menlo

Park, CA 94025, USA

Full list of author information is available at the end of the article
}

(c) The Author(s). 2018 Open Access This article is distributed under the terms of the Creative Commons Attribution 4.0 International License (http://creativecommons.org/licenses/by/4.0/), which permits unrestricted use, distribution, and reproduction in any medium, provided you give appropriate credit to the original author(s) and the source, provide a link to the Creative Commons license, and indicate if changes were made. The Creative Commons Public Domain Dedication waiver (http://creativecommons.org/publicdomain/zero/1.0/) applies to the data made available in this article, unless otherwise stated. 


\section{Background}

Decades of research has demonstrated that cognitive behavioral therapies (CBTs) are effective for a wide variety of psychiatric problems and diagnoses [1]. Given this strong evidence base, CBT is cited as a first-line treatment in treatment guidelines for a variety of mood and anxiety disorders [2, 3] as well as for posttraumatic stress disorder (PTSD) [4, 5]. Numerous national, state, and regional mental health systems are implementing CBTs, requiring clinicians to offer them to their clients as clinically appropriate $[6,7]$. To date, these efforts have largely focused on provider training and outcome monitoring, with less emphasis on ongoing quality assurance $[8,9]$.

Decreases in CBT quality can reduce both consumer access to evidence-based psychosocial treatments (EBPTs) and intended symptom improvement [9]. The possibility of "voltage drops" in effectiveness in non-research settings is therefore of great concern to researchers and policymakers [10]. It is important to monitor treatment quality as well as client characteristics and outcomes to learn whether the treatments are being delivered as intended and to understand reasons for potential differences in effectiveness or engagement [11]. Some research suggests that many providers do not implement EBPTs with fidelity [12-14], and a recent study determined that there was no relationship between self-reported primary cognitive behavioral theoretical orientation and observer-rated CBT competence [15]. In light of such research, ongoing quality monitoring and support is a key component in EBPT implementation and sustainability models [16-19]. Furthermore, articulates, policymakers, healthcare administrators, insurance providers, consumers, and other stakeholders need feasible methods of assessing the quality of services that are being delivered.

Efficient, scalable, and non-invasive methods of identifying what occurs in psychotherapy sessions remain elusive, yet they are critical to supporting and evaluating implementation outcomes. Empirically validated fidelity monitoring and support strategies are not yet in place in most healthcare systems $[4,6,20]$. Instruments developed to rate CBT fidelity are typically based on observation. While considered the "gold standard" in psychotherapy outcome research, observation is time- and labor intensive and not feasible in many practice settings [21]. Furthermore, scalable strategies to understand fidelity in practice is necessary, because for many psychotherapies, the link between observer-rated fidelity to session elements is not entirely clear and may differ based on the population characteristics. A recent meta-analysis demonstrated no overall link between observer-rated fidelity and symptom change across a range of treatments and disorders. Notably, though, in the analyses for many studies included in the meta-analysis, aggregated fidelity across the protocol introduced temporal confounds
[22]. When analyses were conducted to avoid the confound and establish the temporal precedence of fidelity, two aspects of fidelity, adherence to the protocol, and competence (skill of delivery) in early CBT sessions were associated with subsequent decreases in depression [22]. Later research suggested that fidelity to key aspects of the treatment cognitive processing therapy (CPT; a CBT for PTSD), as opposed to prescribed session elements, was associated with symptom change [21, 23]. Thus, research on session fidelity or quality must establish temporal precedence of these factors to understand relationships between fidelity and symptom change in practice and must also examine strategies that emphasize the quality of delivery of critical treatment elements rather than fidelity to session components.

Researchers have also discovered limitations to existing indirect methods of monitoring fidelity, although they are sometimes used for pragmatic reasons [21]. Clinician self-reports of fidelity (e.g., checklists) appear to correspond only modestly with observer or client fidelity ratings $[9,12,15,24-26]$. Both our preliminary work and published literature [27] indicate that clinicians (1) perceive adherence checklists as adding to an already high paperwork burden, (2) may not complete them as directed, and (3) may over- or underreport use of prescribed treatment elements. Literature on medical record review, checklists, and clinical progress notes to assess fidelity is mixed [28], and very little is specific to mental health $[25,28,29]$. One study indicated that record review may be a valid method of assessing clinician behavior [30], but research suggests that clinical progress notes convey poor estimates of frequency or intensity of EBPT techniques [30, 31]. Thus, conclusions about the interventions being delivered, and their quality, that can be drawn from self-report or clinical records may be limited.

While manualized CBTs exist for a wide variety of mental health conditions, a common element is the use of worksheets to teach and support client CBT skill development. Diagnosis-specific and transdiagnostic CBT protocols emphasize a set of core CBT elements, targeting clients' behaviors and thoughts to reduce their symptoms. Both the behavioral and cognitive components of CBTs have been shown to impact symptom change for depression, anxiety, and PTSD [1, 32-35]. Worksheets are used in session to schedule specific behavioral activities and assess their impact on mood, anxiety, or sense of mastery. They are also used to guide one through the processes of cognitive restructuring, which helps clients learn to identify and challenge their maladaptive beliefs and generate balanced alternative thoughts [36, 37]. Assessing the quality of the worksheets that are completed with clinician guidance in sessions may therefore be a pragmatic and valid strategy to assess CBT quality. 
Thus, using worksheets completed in session to assess treatment fidelity may convey advantages over timeintensive observer ratings and imprecise self-reports of session content.

Another advantage of using worksheets to assess fidelity is that CBT worksheets are accessible and easily transmitted. They are available in paper form and through mobile technology that is accessible to people with disabilities (e.g., hearing- or vision-impaired). The majority of adults in advanced economies, and over half in emerging economies, own smartphones [38, 39]. Mobile applications (apps) are increasingly being used in CBT sessions, making transmission for real-time quality assessment feasible. Worksheets have been adapted for lower levels of literacy, multiple languages, traumatic brain injury, and other factors. Thus, it is possible to tailor worksheets that fit client needs and tailor strategies for collection and rating for different settings. However, the quality and feasibility of data collection may vary across formats and settings. For example, app worksheets may be more legible than handwritten data but may also be briefer or less detailed due to difficulties in typing on a phone. We propose to refine and test a unique method of assessing treatment quality through worksheets generated through routine clinical procedures. Specifically, we aim to (1) develop a scalable general CBT quality measure based on work samples rather than self-report or observation and (2) to evaluate the reliability, validity, and feasibility of different strategies for collecting these materials (e.g., mobile apps vs. paper worksheets).

\section{Preliminary work}

\section{Research on worksheet quality}

In a preliminary study, we developed a method to assess fidelity to CPT by coding the level of adherence and competence reflected in worksheets that were completed in treatment sessions [40].

\section{Reliability}

Inter-rater reliability was high (ICC $=.68$ to .90$)$ for clinician competence on different worksheets. It was adequate to almost perfect (ICC $=.55$ to .92$)$ on ratings of client skill in completing worksheets independently.

\section{Convergent validity}

Observer-rated competence on full sessions were not significantly correlated with worksheet ratings $\left(r_{p b}=.278\right.$, $p=.068$ ), but we found high (Spearman rank) correlations between our worksheet rating method and observer ratings for worksheet-related CBT elements (e.g., cognitive restructuring, $\left.r_{p b}=.823 ; p=.000\right)$. Similarly, correlation between observer-rated adherence ( $n=44$ sessions) and adherence scores based on worksheets was low for full sessions $\left(r_{p b}=.062, p=.68\right)$ but high for worksheet-related items $\left(r_{p b}=.808 ; p=.000\right)$.

\section{Association with symptom change}

Overall, our preliminary findings suggest that the worksheet scores were uniquely associated with subsequent symptom change, but client skill was not. Additionally, our preliminary work indicated that symptoms do not predict the quality of worksheets completed in session, although they did predict the quality of worksheets done for homework without clinician guidance [40].

\section{Relative advantage}

Raters recorded the time to complete ratings (one or more worksheet per session). The mean was $7.04 \mathrm{~min}$ $(\mathrm{SD}=3.90)$. Observation and rating of a full session requires 60-75 min or more per session, meaning that this strategy requires approximately $10 \%$ of the time required for "gold standard" ratings. The measure had good face validity, and raters viewed worksheets as moderately easy to rate.

\section{Methods \\ CBT worksheet scoring development}

Following an iterative process used in previous study, the team's subject matter experts (who have knowledge of both CBT and the systems in which it is implemented) will develop and refine the scoring system developed in the preliminary study to generalize to common CBT worksheets, with input from end-users (e.g., clinicians, administrators, policymakers, supervisors). We will collect CBT worksheets that are distributed by large mental health systems and CBT training programs and publicly or commercially available books and manuals to identify common elements of CBT worksheets. We will use a rational approach to measure construction and refinement [41, 42], following methods outlined by Vogt and colleagues [35, 43]. We will create a brief, content-saturated measure of CBT quality, with conceptually distinct $\mathrm{CBT}$ domains.

We anticipate that minimal changes will be required to make the scoring system developed in the preliminary study applicable to CBT worksheets related to cognitive restructuring, given their similarity to $\mathrm{CPT}$ worksheets. Nearly all worksheets that focus on cognitive restructuring contain the following elements: identification of a situation, thought, and feeling/response, questions to challenge beliefs (e.g., evidence for and against), maladaptive thinking patterns, a new/balanced belief, and intensity rating of the emotions and beliefs before and after the cognitive restructuring process. We will develop scoring for common elements of worksheets that track or plan situations, behaviors, emotions, thoughts, and associated intensity ratings. 
During the development phase, we will use worksheets collected in previous research. Our team will score these worksheets, and end-users (e.g., clinicians, CBT trainers, quality monitors, administrators, supervisors) will provide feedback on clarity of items, scoring rules, and ease of use, which will help us refine the measure.

Only worksheets that were completed or reviewed and refined in session will be scored to assess CBT quality. Raters will choose items from a list of domains that reflect the instructions and questions on the worksheet to be rated. Items will be scored on a Likert-type scale, and a mean score will be calculated based on all applicable worksheet items. Session scores will be a mean of all worksheets that were completed in session.

We will next examine test-retest reliability, rater agreement on the measure, and the correlation between worksheet ratings and observer ratings of fidelity. We will conduct preliminary analyses to assess associations with symptom change. As we refine the measure and identify content domains, items with higher item-total correlations will take precedence over those with lower item-total correlations, and we will take user feedback into account as well as we refine the measure before entering the validation phase.

\section{Data collection and research strategy summary}

After the development of the scoring system, we will prospectively collect data in a variety of settings, with clinicians $(n=120)$ who have varying levels of experience and expertise with CBT. We will collect data on clinician, client, and organizational characteristics that may impact the assessment of psychotherapy quality. We will collect worksheets, symptom measures, clinical notes with checklists of CBT strategies embedded within them, and therapy session recordings for participating clients. They will be provided to the investigators using secure, Institutional Review Board (IRB)-approved modes of data transmission that have been used successfully in our previous research [44-47].

Participating clients will be randomized into one of two different worksheet formats-app version or paper form (content is the same, mode of completion and data transmission differs). Raters will access the copies of the worksheets, or the mobile app dashboard, and score the worksheets. Clinicians, administrators, supervisors, and clients will be interviewed to assess perceptions of the quality assessment strategies and to determine feasibility, and these data will be analyzed using qualitative strategies.

The variety of worksheets and data capture sources (apps, paper forms) will allow assessment of clinician and client preferences, the degree of detail and completion required for accurate quality assessment. This strategy will allow us to answer several questions through our statistical and qualitative analyses. Analyses will be conducted to answer questions and test hypotheses regarding (1) the reliability and factor structure of the measure, (2) associations between the quality measure and subsequent symptom change (the primary outcome), (3) the concurrent validity of the measure, (4) whether the measure performs better than adherence checklists embedded in clinical notes, (5) whether different formats (apps vs. paper forms) have different concurrent or predictive validity, and (6) the feasibility, burden, satisfaction, and time requirements for the worksheets vs. the adherence checklists.

\section{Data collection}

We will collect worksheets and clinical note templates (which contain adherence checklists) from clinicians who have varying degrees of CBT expertise in a variety of settings The data sets will include variability in population characteristics and clinician training/education level.

\section{Clinician inclusion criteria}

The clinician inclusion criteria are as follows: (1) be trained (workshop or web-based training and consultation) or in training for CPT for PTSD or CBT that uses worksheets, (2) anticipate at least three eligible clients, (3) be willing to record sessions and provide worksheets and symptom measures to the study, (4) have computer and Internet access, and (5) be willing to use a mobile app on a tablet or mobile device.

\section{Client inclusion criteria}

Eligible clients $(n=360)$ will be $(1)$ adult outpatients (ages 18 or older), with a clinician diagnosis of primary PTSD (PTSD-Checklist-5 score of 33 or above) [48], a depressive disorder (e.g., major depressive disorder, dysthymia; Patient Health Questionnaire score of 10 or above) [49], or an anxiety disorder (Beck Anxiety Inventory score 22 or above) [50, 51]; (2) those who are willing to allow the team to collect session recordings, measures, notes, and worksheets; (3) those who are able to read and write at a 6th-grade level or above; and (4) those who are willing to engage in CBT.

\section{Client exclusion criteria}

The client exclusion criteria are as follows: (1) imminent risk for suicide or homicide (requiring hospitalization), (2) in need of detoxification (can be enrolled when substance abuse treatment is not the primary treatment target), (3) active psychosis or manic episode, or (4) cognitive impairments that preclude any participation in therapy. 


\section{Procedure}

\section{Recruitment and informed consent}

Clinicians will be recruited through previously used recruitment strategies including presentation at clinical team meetings (at participating clinics), at CBT trainings, and/or by email. Private practitioners will be targeted through emails to professional organization listservs. After the informed consent, they will complete the clinician measures. They will be provided with an iPod touch, if needed, to use the mobile app. Their clients will be recruited using strategies approved by local systems and IRBs, such as waiting room flyers, whiteboard videos, and/ or clinician or intake coordinator referral. Informed consent will be completed by study staff. Clients will then be randomized into one of two worksheet completion strategies: mobile app vs. paper form. Their sessions will be audio-recorded, worksheets will be collected, and they will complete symptom measures (every session for primary problem, every fourth for additional measures) for up to 16 sessions.

\section{Worksheet transmission}

Clinicians will redact any potential identifiers from worksheets and indicate whether they were completed/ reviewed in session or for homework. Paper worksheets will be transmitted by fax or scanned and transmitted electronically (e.g., secure email, shared network drives, and HIPAA-compliant data storage software) depending on local capacity and regulations. The mobile apps will record the scheduled session time and timestamp the worksheets to allow discernment of whether worksheets were done in session. We will use Qualtrics to develop app-based standard cognitive and behavioral worksheets used for depression, anxiety, and PTSD. We will only score worksheets that are done in session to measure quality; scoring of worksheets that are completed for homework will be used to covary client skill in analyses.

\section{Collection of adherence checklists}

Because templated clinical notes that include checklists of CBT strategies are mandated in some systems and because an alternative hypothesis is that such notes may be an even more feasible, lower-burden strategy for assessing quality, particularly when notes are electronic, we will also collect clinical notes. If extraction from medical records is not possible, copies will be collected in the same way as the paper worksheets and/or using Qualtrics.

\section{Collection of session audio}

Sessions will be digitally recorded and uploaded to secure servers and HIPAA-compliant data software systems. These strategies have been successful in our previous research, in which over 600 clinicians have provided sessions for full CPT and CBT protocols [45, 52-54].
Clinicians provided similar data when originally trained in the treatments $[3,55,56]$.

\section{Incentives and retention}

Clinicians will receive their worksheet ratings at the end of the study; these will include text with bullet-pointed suggestions for improvement. Clinic-level incentives such a breakfast or lunch tray will be provided once per year, if clinicians keep up with benchmarks for providing data (e.g., $85+\%$ of required data for currently enrolled clients). If their organization permits, they will receive a gift card for the time they spend transmitting client data (e.g., session recordings, worksheets, symptom measures) for each client. Clients will receive a gift card, if they complete at least four sessions and complete their measures for these sessions.

\section{Measures and assessment strategy Outcomes and potential mediators}

Clients will complete the measure for their primary problem/disorder each session (standard CBT practice). Clients with depression disorder will complete the Patient Health Questionnaire-9 (PHQ-9); clients with anxiety will complete the Beck Anxiety Inventory (BAI) each session, and clients with PTSD will complete the PTSD checklist-5 (PCL-5) every session [48, 57, 58]. We will standardize scores when examining outcomes for analyses but will also look separately at depression, anxiety, and PTSD outcomes in our analyses. Depression, anxiety (PHQ-9 and/ or BAI, when not the primary target for treatment), and functioning (Brief Inventory for Psychosocial Functioning; B-IPF) [59] will be measured at the fourth session and posttreatment.

\section{Client variables and measures}

Secondary analyses will explore whether the rating method is less accurate for subsets of the population. We considered brief tests of literacy, English proficiency, or executive functioning, but because they are orally administered and require assessor training, they are not feasible for clinicians to administer and may cause client discomfort. Instead, we will use variables that capture related and correlated factors including traumatic brain injury and comorbidity (via clinician report) and other demographics via self-report.

\section{Client skill}

As with our previous work, we will calculate scores for client skill (ability to complete homework sheets; client worksheets will be rated on the same measure). We will report the range of abilities of our sample and use the scores as a covariate in analyses of the association between quality and outcomes. 


\section{CBT fidelity}

We will use the Cognitive Therapy Rating Scale (CTRS) $[60,61]$, the "gold standard" observer rating scales for CBT for all sessions, and for CPT sessions, we will also use the CPT observer rating scale [21]. Trained postdoctoral fellows and advanced graduate students will rate $10 \%$ of the session recordings. They will overlap on $10-15 \%$ of these sessions to facilitate reliability analyses. ICCs for observer ratings in our previous studies using these instruments have been high, ranging from .79 to .84 ([45]; Monson et al.: A randomized controlled trial of training and consultation methods to deliver cognitive processing therapy for posttraumatic stress disorder: Impact on patient outcomes, submitted).

\section{Clinician and organization variables: covariates}

Additional measures will be collected at baseline to explore organization- and clinician-level moderating effects on the association between quality on clinical outcomes (Clinician Demographic Characteristics and Experience Questionnaire, Evidence-Based Practice Attitudes Scale, and Perceived Chrematistics of Interventions and the Implementation climate Assessment) [62-64]. We will assess characteristics that may contribute to quality outcomes [65]: degree, years of experience, gender, age, and prior $\mathrm{CBT} / \mathrm{EBPT}$ training.

\section{Interviews and assessment of stakeholder perspectives}

We will use brief rater surveys and stakeholder interviews (raters, clinicians, 15-20 clients per system, policymakers, and administrators) in each system to assess relevant constructs of the Consolidated Framework for Implementation Research (CFIR) [66] such as perceptions of relative advantage, complexity, design quality/ packaging, readiness for implementation, and inner and outer context factors that might influence implementation. We will assess time required for rating, identify roles that would most likely serve as quality assessors in the different systems (e.g., supervisor, quality assurance staff), assess hourly costs associated with each clinical/quality assessor role, and assess time required for transmitting the worksheets for app vs paper formats. We will assess productivity demands and other demands and responsibilities that may impact use of the assessment strategies. We will follow up to assess perspectives on the contribution of client, clinician, and system characteristics to any variance in quality scores that is identified in our analyses. We will also assess perspectives on use of the mobile apps vs. paper forms, satisfaction, suggestions for refinement, and perceived adequacy of information in the quality measures.

\section{Raters and scoring}

We will apply the rating system that is refined in the first phase of the study to worksheets that are collected during the entire course of the study. The scoring rules (including decision rules for each session) and scoring algorithms are embedded in an electronic database for app and paper copies. The cadre of raters will include postdoctoral fellows from each system and bachelor's level research assistants, and some are able to rate sessions and worksheets in Spanish. They will be trained to use the measure and to become familiar with all decision rules, and new decision rules may be identified during this training process. These sessions will be evenly distributed between raters. A subset (10\%) will be rated by a second time (1 week after the first rating) to assess test-retest reliability. Furthermore, participating systems will nominate one person (whoever would be in a position to monitor quality) to receive brief training and rate 10 sessions to determine feasibility of training system/clinic-level quality monitors. They will be interviewed regarding potential for implementation within their setting. A randomly selected subset $(20 \%)$ of the worksheets will be rated by CBT experts and compared with our raters. To score the adherence checklists embedded in the clinical notes, we will score the proportion of CBT elements that are checked off by clinicians.

\section{Analytic strategy}

We will conduct the following analyses:

\section{Reliability}

We will examine correlations between the subset of worksheets that are rated twice to examine test-retest reliability. Next, we will compute estimates of internal consistency reliability for hypothesized CBT domains (e.g., identifying thoughts and feelings, cognitive restructuring, behavior planning and tracking) in the form of Cronbach's alphas and retain domains with an alpha of .70 or above. We will calculate intraclass correlations (ICCs) for the total measure and for each content domain, using a random effects model to estimate the reliability of rater judgments [67]. Per established conventions and prior research [68], we will apply a minimum criterion ICC $=.60$ ("good") [69] to indicate acceptable agreement. We will also compare bachelor's level raters' scores to expert rater scores to assess the degree of agreement, to determine a level of expertise required for rating.

\section{Concurrent validity}

We will compare our strategy to the "gold standard" fidelity measure and clinical progress notes alone. We predict that ratings on our quality monitoring method will be highly correlated with existing $[3,17]$ objective and reliable observer ratings and that they will be more accurate than the ratings based on the checklists embedded in the clinical notes alone [2, 21, 70, 71]. We will examine Spearman rank correlations [72] between our 
method and independent observer ratings for the overall measure, for each subscale and for conceptually corresponding items. Further, we will conduct cross-validation borrowing the idea of checking internal validity of prediction models in statistical learning [73].

\section{Factor analysis}

We will conduct an exploratory factor analysis to examine the factor structure of items within each worksheet. It is possible that quality may differ across different elements/domains of CBT, but it is also possible that CBT quality is better represented as a composite variable (some areas are of higher quality than others and that summing across them still provides good information about overall quality). Thus, we will explore the possibility of multiple factors or domains (e.g., cognitive restructuring), and will also evaluate the extent to which the components are best represented as a latent variable, that is, a general tendency to provide high-quality CBT that is reflected in high scores across different CBT components.

\section{Clinical outcome predictions}

We expect that clients whose sessions receive higher quality ratings will exhibit larger decreases in symptoms compared to clients whose sessions had lower ratings. Based on previous research suggesting that it is important to assess fidelity in early sessions to avoid temporal confounds between process variables and outcomes $[22,68,74,75]$, we expect that quality in earlier sessions will more accurately reflect the contributions of session quality and clinician skill (rather than client factors) than that in later sessions. Primary analyses will focus on quality in sessions 1-6. We will cross-validate prediction models as a way of checking internal validity (generalization error).

\section{Analyses}

We will first examine distribution of key variables for skewness, variability, and outliers and apply appropriate transformation or other strategies to address nonnormality (e.g., robust estimation procedures) as necessary. Symptom scores from instruments that reflect the target problem for each client (e.g., depression, anxiety, or PTSD) will be standardized, and the standard scores will be used as the dependent variable (DV). We will also conduct secondary analyses with the measures for each target problem and with the functioning measure as DVs. The study will produce hierarchical data with repeated measures (Level-1) nested within clients (Level-2) nested within clinicians (Level-3). To evaluate change in client outcomes, we will conducted multilevel regression (i.e., mixed-effects regression, hierarchical linear modeling) growth curve analysis, which offers numerous strengths for analyzing change in nested data, including efficiency in handling missing data, powerful and accurate estimation procedures adjusting for clustering, and modeling flexibility (e.g., allows for the inclusion of continuous or categorical, time-invariant or time-varying covariates and predictors). We will employ strategies to check the sensitivity of analysis results due to missing data (e.g., pattern mixture modeling) [76]. We will analyze the intent-to-treat sample [77] and completer samples. First, multiple unconditional change models (i.e., change without predictors) will be evaluated to determine the most reliable and powerful way to analyze change (e.g., linear or non-linear change; modeling time as time since baseline or as session number) and determine the most appropriate variance-covariance structure, considering, among others, the autoregressive structure.

The first set of multilevel regression analyses will evaluate trajectories of outcomes over the entire course of therapy or over the course of several sessions. We will examine the influence of potentially significant covariates (e.g., baseline scores, setting, veteran status, client skill, and other clinician, setting, or client characteristics) and include in exploratory analyses. Given the small number of systems, the system will be entered as a potential covariate to assess and adjust for its influence, but we will conduct an exploratory analysis with the system entered at Level-4.

The next multi-level regression analyses will examine the session-by-session associations between quality and symptoms. Lagged multivariate models will allow for the inclusion of two outcomes (quality and symptom change) in the same model [78-80]. Evaluation of the cross-lagged paths will provide information about temporal precedence in the relationship between the two variables across time. We will also account for overall increases and decreases in the model by including time as a predictor of each outcome.

We will next determine whether the different quality measures or worksheet formats differ in terms of how strongly they are associated with subsequent symptom change. Since clients will be randomized into worksheet format (app vs paper form), a dichotomous variable with paper form as the reference will be entered at Level-2 in the analyses described above to examine whether format predicts symptom change. We will explore potential interactions with target problem (PTSD, depression, anxiety). We will examine and compare the effect sizes and the proportion of variance accounted for by the worksheet measure and the adherence checklist. We will also compare them for app vs. paper form worksheets. A medium or large effect would be clinically meaningful in the context of the pragmatic goals of this project, particularly because the reliable change index for each of our outcome measures corresponds to a large effect size $[48,49,58]$. On the other hand, if associations with outcomes differ only slightly, we would recommend selection of a quality assessment strategy based on specific programs' needs or goals. 
Comparisons of quality measures (worksheet vs. adherence checklists) and worksheet formats

If self-reported adherence (as assessed through the checklists embedded in clinical notes) is highly correlated with worksheets and/or the gold standard observer ratings, and if they are strongly associated with outcomes, the checklists alone may be deemed sufficient for quality assessment, because the notes can already be accessed by quality monitors in each system. First, we will use the Fisher $r$-to- $z$ transformation to calculate a value of $z$ that can be applied to assess the significance of the difference between two correlation coefficients. This analysis is conducted to assess whether a significant difference exists between correlations with the gold standard observer ratings. If there is no significant difference, this would indicate that the worksheet quality scoring strategy does not yield an advantage or disadvantage in terms of its correspondence with gold standard observer ratings. We will also use this strategy to determine whether a difference exists between correlations with worksheet formats (app vs. traditional paper form) and observer ratings [48].

\section{Sample size justification and power calculations}

In light of the project's emphasis on the development of pragmatic measurement strategies that predict changes in the target problems, we powered the study to test for predictive validity. Calculations of a design effect (measure of how the design effects the standard error of the parameters) [81, 82] accounted for clustering. We computed ICC with repeated observations of clients at the clinic $\left(r_{p b}=.01\right)$, clinician $\left(r_{p b}=.01\right)$, and client $\left(r_{p b}=.10\right)$ level in our prior research, assuming three clients per clinician, and six clinicians per cluster, and six observations per client, yielding a design effect of up to 2.04. We based the estimated number of observations per client to conservatively account for potential 37\% client-level attrition and missing data during the treatment. For the growth curve analyses to assess whether the quality measure predicts symptom change (our primary analysis), the probability of a type 1 error (alpha) at two-tailed .05 power exceeds .80 for an effect size of $r=.30$, which corresponds with small-tomedium effects of fidelity on symptoms in prior CBT fidelity research $[68,83]$. We also projected a sample size to be sufficient to conduct separate analyses and test for interactions with disorder (PTSD vs. depression vs. anxiety), app vs. paper form, and other variables of interest, and detect an effect size of .50, a medium effect.

\section{Discussion}

To date, there is no feasible and easily adoptable way to effectively assess clinician fidelity of CBTs. This study aims to refine and test a unique method of leveraging data generated through routine clinical procedures to assess quality to (1) develop a scalable general CBT quality measure based on work samples rather than self-report or observation and (2) to evaluate the reliability, validity, and feasibility of different strategies for collecting these materials. Our method poses little additional burden to clients or clinicians. Because it uses routine clinical materials, it allows for a less "invasive" review of a random selection of sessions by a quality monitor or supervisor. Since treatments are sometimes modified in practice settings [8], it will be designed to apply to modifications to worksheets that are adapted to meet client needs. To our knowledge, this will be the first instrument of this nature to be developed and validated. It will thus fill a critical gap in the field and has the potential to make large-scale quality monitoring in research and practice settings more feasible and efficient. Scalable methods of quality monitoring are vital to efforts to study and promote the implementation of EBPTs [84, 85]. This study has strong potential to impact fidelity monitoring strategies for a variety of CBTs due to shared elements across CBTs [36]. A less burdensome method can dramatically increase feasibility of ongoing quality monitoring. Validation across platforms (mobile app and traditional paper forms) and data on the feasibility and acceptability of each of these platforms is an additional innovation that will yield actionable data to inform implementation. This in turn can promote continued consumer access to highquality delivery of CBT and other EBPTs [9, 16, 22], and protect the significant investment in implementation across multiple systems.

\section{Abbreviations}

app: Application; BAl: Beck's anxiety inventory; CBT: Cognitive behavioral therapy; CPT: Cognitive processing therapy; CTRS: Cognitive therapy rating scale; EBPT: Evidence-based psychosocial treatment; HIPAA: Health Insurance Portability and Accountability Act; ICC: Intraclass correlations; IRB: Institutional review board; PCL-5: Posttraumatic stress disorder checklist-5; PHQ-9: Patient health questionnaire-9; PTSD: Posttraumatic stress disorder; SD: Standard deviation; VA: Veterans affairs

\section{Acknowledgements}

We would like to acknowledge the postdoctoral fellows and project staff who have contributed to or are contributing to the project development and operations including Nicholas Affrunti, PhD, Soo Jeong Youn, PhD, Kaylie Patrick, Emily Ahles, Yesenia Aguilar Silvan, Caroline Canale, Danielle Centeno, Jansey Lagdamen, Katlyn Welch, Kiley Dunlap, and Patricia Carreño. We also would like to gratefully acknowledge the contributions and efforts of the organizations in the community that have partnered with us in this research effort.

\section{Funding}

This project is supported by the National Institute of Mental Health, funding reference number R01MH112628-01. The pilot work was supported by funding reference number: R21MH097022.

Availability of data and materials

Data is being collected for this study. Upon completion of the study, resources produced through this research (manuals, scoring systems) will be made available. 


\section{Authors' contributions}

The study was conceptualized by SWS, LM, CC, and TC with input from RD, PB EK, JO, DV, MS, JB, and SS. Suggestions for refinement of study procedures and assessments were made by $\mathrm{CJ}, \mathrm{KM}$, and HLB. All authors read and approved the final manuscript.

\section{Ethics approval and consent to participate}

This study was approved by the institutional review board at the Stanford University and VA Palo Alto Healthcare System, Palo Alto, CA, the City of Philadelphia Department of Public Health Institutional Review Board, Philadelphia, PA, the institutional review board at VA Boston Healthcare System and Boston University, the institutional review board of Massachusetts General Hospital and the Partners Human Research Committee, Somerville, MA, and the institutional review board at University of Pennsylvania, Philadelphia, PA.

\section{Competing interests}

The authors declare that they have no competing interests.

\section{Publisher's Note}

Springer Nature remains neutral with regard to jurisdictional claims in published maps and institutional affiliations.

\section{Author details}

'National Center for PTSD, VA Palo Alto HCS and Stanford University Department of Psychiatry and Behavioral Sciences, 795 Willow Road, Menlo Park, CA 94025, USA. ${ }^{2}$ Harvard Medical School and Massachusetts General Hospital, 70 Everett Ave., Chelsea, MA 02150, USA. ${ }^{3}$ University of Pennsylvania, Perelman School of Medicine, University of Pennsylvania, 3535 Market Street, Suite 3038, Philadelphia, PA 19104, USA. ${ }^{4}$ National Center for PTSD, VA Boston Healthcare System and Boston University School of Medicine, 150 S. Huntington Ave., Boston, MA 02130, USA. ${ }^{5}$ School of Arts and Sciences, University of Pennsylvania, 425 S. University Ave., Philadelphia, PA 19104, USA. 'Palo Alto Veterans Institute for Research, 3801 Miranda Ave., Palo Alto, CA 94304, USA. ${ }^{7}$ Suffolk University, 73 Tremont Street, Boston, MA 02108, USA. ${ }^{8}$ Stanford University, 401 Quarry Rd, Stanford, CA 94305, USA. ${ }^{9}$ Oregon Social Learning Center, Eugene, OR 97401, USA. ${ }^{10}$ National Center for PTSD, 795 Willow Road, Menlo Park, CA 94025, USA.

\section{Received: 29 March 2018 Accepted: 19 April 2018}

\section{Published online: 22 May 2018}

\section{References}

1. Beck AT. The current state of cognitive therapy: a 40-year retrospective. Arch Gen Psychiatry. 2005;62(9):953-9.

2. Clark DM. Implementing NICE guidelines for the psychological treatment of depression and anxiety disorders: the IAPT experience. Int Rev Psychiatry. 2011;23(4):318-27.

3. Karlin BE, Cross G. From the laboratory to the therapy room: national dissemination and implementation of evidence-based psychotherapies in the US Department of Veterans Affairs Health Care System. Am Psychol. 2014;69(1):19-33.

4. Karlin BE, Ruzek Jl, Chard KM, Eftekhari A, Monson CM, Hembree EA, Resick PA, Foa EB. Dissemination of evidence-based psychological treatments for posttraumatic stress disorder in the veterans health administration. Trauma Stress. 2010;23(6):663-73.

5. Foa EB, Keane TM, Friedman MJ, Cohen JA. Effective treatments for PTSD: practice guidelines from the International Society for Traumatic Stress Studies. 2nd ed. New York, NY: Guilford Press; 2009.

6. McHugh RK, Barlow DH. The dissemination and implementation of evidence-based psychological treatments: a review of current efforts. Am Psychol. 2010;65:73-84

7. Stirman SW, Buchhofer R, McLaulin JB, Evans AC, Beck AT. The Beck initiative: a partnership to implement cognitive therapy in a community behavioral health system. Psychiatr Serv. 2009;60:1302-10.

8. Mendel P, Meredith LS, Schoenbaum M, Sherbourne CD, Wells KB. Interventions in organizational and community context: a framework for building evidence on dissemination and implementation in health services research. Adm Policy Ment Health Ment Health Serv Res. 2008;35(1):21-37.

9. Schoenwald SK. It's a bird, it's a plane, it's ... fidelity measurement in the real world. Clin Psychol Sci Pract. 2011;18(2):142-7.
10. Chambers DA, Glasgow RE, Stange KC. The dynamic sustainability framework: addressing the paradox of sustainment amid ongoing change. Implement Sci. 2013;8(1):117.

11. Schoenwald SK, Garland AF, Chapman JE, Frazier SL, Sheidow AJ, SouthamGerow MA. Toward the effective and efficient measurement of implementation fidelity. Adm Policy Ment Health Ment Health Serv Res. 2011;38(1):32-43.

12. Santa Ana EJ, Martino S, Ball SA, Nich C, Frankforter TL, Carroll KM. What is usual about 'treatment-as-usual'? Data from two multisite effectiveness trials. J Subst Abus Treat. 2008;35(4):369-79.

13. Stirman SW, Kimberly JR. Monitoring fidelity in longitudinal implementation research. Bethesda, MD: 2nd annual NIH Conference on the Science of Dissemination and Implementation: 2009; 2009.

14. Stirman SW, Kimberly JR, Calloway A, Cook N, Castro F, Charns MP. The sustainability of new programs and interventions: a review of the empirical literature and recommendations for future research. Implement Sci. 2012;7:17.

15. Creed TA, Wolk CB, Feinberg B, Evans AC, Beck AT. Beyond the label: relationship between community therapists' self-report of a cognitive behavioral therapy orientation and observed skills. Admin Pol Ment Health. 2016;43(1):36-43.

16. Aarons G, Hurlburt M, Horwitz SM. Advancing a conceptual model of evidence-based practice implementation in public service sectors. Adm Policy Ment Health Ment Health Serv Res. 2011;38(1):4-23.

17. Shediac-Rizkallah MC, Bone LR. Planning for the sustainability of communitybased health programs: conceptual frameworks and future directions for research, practice and policy. Health Educ Res. 1998;13(1):87-108.

18. Racine DP. Reliable effectiveness: a theory on sustaining and replicating worthwhile innovations. Admin Pol Ment Health. 2006:33(3):356-87.

19. Feldstein $A C$, Glasgow RE, Smith DH. A practical, robust implementation and sustainability model (PRISM) for integrating research findings into practice. Joint Comm J Qual Patient Saf. 2008;34(4):228-43.

20. Wells KB, Miranda J. Promise of interventions and services research: can it transform practice? Clin Psychol Sci Pract. 2006;13(1):99-104.

21. Nishith P, Resick PA. Cognitive processing therapy (CPT): therapist adherence and competence protocol. St. Louis: Unpublished manuscript, University of Missouri; 1994.

22. Webb CA, Derubeis RJ, Barber JP. Therapist adherence/competence and treatment outcome: a meta-analytic review. J Consult Clin Psychol. 2010; 78(2):200-11.

23. Farmer CC, Mitchell KS, Parker-Guilbert K, Galovski TE. Fidelity to the cognitive processing therapy protocol: evaluation of critical elements. Behav Ther. 2017:48(2):195-206.

24. Carroll KM, Martino S, Rounsaville BJ. No train, no gain? Clin Psychol Sci Pract. 2010;17(1):36-40.

25. Decker SE, Jameson MT, Naugle AE. Therapist training in empirically supported treatments: a review of evaluation methods for short- and long-term outcomes. Adm Policy Ment Health Ment Health Serv Res. 2011;38(4):254-86.

26. Perepletchikova F, Treat TA, Kazdin AE. Treatment integrity in psychotherapy research: analysis of the studies and examination of the associated factors. J Consult Clin Psychol. 2007;75(6):829-41.

27. Kauth MR, Sullivan G, Blevins D, Cully JA, Landes RD, Said Q, Teasdale TA. Employing external facilitation to implement cognitive behavioral therapy in VA clinics: a pilot study. Implement Sci. 2010;5(75):1-11.

28. Hrisos S, Eccles M, Francis J, Dickinson H, Kaner E, Beyer F, Johnston M. Are there valid proxy measures of clinical behaviour? A systematic review. Implement Sci. 2009;4:37.

29. Eccles M, Hrisos S, Francis J, Kaner E, Dickinson H, Beyer F, Johnston M. Do self-reported intentions predict clinicians' behaviour: a systematic review. Implement Sci. 2006;1(1):28.

30. Liddle HA, Rowe C, Gonzalez A, Henderson CE, Dakof G, Greenbaum P. Changing provider practices, program environment, and improving outcomes by transporting multidimensional family therapy to an adolescent drug treatment setting. Am J Addict. 2006;15:102-12.

31. Jensen-Doss A, Cusack KJ, de Arellano M. Workshop-based training in trauma-focused CBT: an in-depth analysis of impact on provider practices. Community Ment Health J. 2008:44:227-44.

32. Jacobson NS, Dobson KS, Truax PA, Addis ME, Koerner K, Gollan JK, Gortner E, Prince SE. A component analysis of cognitive-behavioral treatment for depression. J Consult Clin Psychol. 1996;64(2):295.

33. Acierno R, Gros DF, Ruggiero KJ, Hernandez-Tejada BMA, Knapp RG, Lejuez CW, Muzzy W, Frueh CB, Egede LE, Tuerk PW. Behavioral activation and 
therapeutic exposure for posttraumatic stress disorder: a noninferiority trial of treatment delivered in person versus home-based telehealth. Depress Anxiety. 2016;33(5):415-23.

34. Keefe JR, Webb CA, DeRubeis RJ. Cognitive therapy for depression, early focus on maladaptive beliefs may be especially efficacious for patients with personality disorders. J Consult Clin Psychol. 2016;84(4):353-64.

35. Vogt D, Shipherd JC, Resick PA. Posttraumatic maladaptive beliefs scale: evolution of the personal beliefs and reactions scale. Assessment. 2012; 19(3):308-17.

36. Evans-Jones C: The CBT handbook: cognitive behavior therapy Speechmark; 2010.

37. Resick PA, Nishith P, Griffin MG. How well does cognitive-behavioral therapy treat symptoms of complex PTSD? An examination of child sexual abuse survivors within a clinical trial. CNS Spectrums. 2003;8:340-55.

38. Ben-Zeev D, Davis KE, Kaiser S, Krzsos I, Drake RE. Mobile technologies among people with serious mental illness: opportunities for future services. Adm Policy Ment Health Ment Health Serv Res. 2013;40(4):340-3.

39. Poushter J. Smartphone ownership and internet usage continues to climb in emerging economies. Pew Research Center. 2016;22:1-44.

40. Wiltsey Stirman S, Gutner C, Gamarra J, Vogt D, Suvak M, Wachen J, Dondanville K, Yarvis J, Mintz J, Peterson A, et al. Leveraging routine clinical materials to assess fidelity to CPT. In: Proceedings of the 3rd Biennial Conference of the Society for Implementation Research Collaboration (SIRC) 2015: advancing efficient methodologies through community partnerships and team science. Implement Sci. 2016;11(1):1.

41. Jackson DN. The dynamics of structured personality tests. Psychol Rev. 1971; 78:229-48.

42. Nunnally JC. Psychometric theory 2nd edn. New York: McGraw-Hill; 1978

43. Vogt $D$, Colvin R. Assessment of accurate self-knowledge. J Pers Assess. 2005;84(3):239-51.

44. Valentine SE, Borba CP, Dixon L, Vaewsorn AS, Guajardo JG, Resick PA, Wiltsey Stirman S, Marques L. Cognitive processing therapy for Spanishspeaking Latinos: a formative study of a model-driven cultural adaptation of the manual to enhance implementation in a usual care setting. J Clin Psychol. 2017;73(3):239-56.

45. Creed TA, Frankel SA, German RE, Green KL, Jager-Hyman S, Taylor KP, Adler AD, Wolk CB, Stirman SW, Waltman SH, Williston MA. Implementation of transdiagnostic cognitive therapy in community behavioral health: The Beck Community Initiative. J Consult Clin Psychol. 2016;84(12):1116.

46. Stirman SW, Pontoski K, Creed TA, Xhezo R, Evans AC, Beck AT, CritsChristoph P. A non-randomized comparison of strategies for consultation in a community-academic training program to implement an evidence-based psychotherapy. Adm Policy Mental Health Serv Mental Health Serv Res. 2017:44(1):55-66.

47. Stirman SW, Shields N, Deloriea J, Landy MSH, Belus JM, Maslej MM, Monson CM. A randomized controlled dismantling trial of post-workshop consultation strategies to increase effectiveness and fidelity to an evidencebased psychotherapy for posttraumatic stress disorder. Implement Sci. 2013; 8(82):1-8.

48. Weathers FW, Litz BT, Keane TM, Palmieri PA, Marx BP, Schnurr PP: The PTSD checklist for DSM-5 (PCL-5). In: Scale available from the National Center for PTSD website. 2010. https://www.ptsd.va.gov/professional/assessment/adultsr/ptsd-checklist.asp. Accessed 5 Dec 2017

49. Beck AT, Steer RA, Ball R, Ranieri WF. Comparison of Beck Depression Inventories-IA and -II in psychiatric outpatients. J Pers Assess. 1996;67(3): 588-97

50. Beck AT, Steer RA. Manual for the Beck anxiety inventory. 1990.

51. Beck AT, Steer RA. Beck hopelessness scale. Psychological Corporation San Antonio; 1988

52. Stirman SW, Shields N, Deloriea J, Landy MS, Belus JM, Maslej MM, Lane J, Monson CM. A randomized comparison of three post-workshop consultation strategies. Philadelphia, PA: Association for Behavioral and Cognitive Therapies: 2014; 2014.

53. Marques L, Eustis EH, Dixon L, Valentine SE, Borba C, Simon NM, Kaysen D, Wiltsey-Stirman S. Delivering cognitive processing therapy in a community health setting: the influence of Latino culture and community violence on posttraumatic cognitions. Psychol Trauma: Theory Res, Pract, Policy. 2016;9:98-106.

54. Gutner C, Barlow D, Sloan D, Wiltsey Stirman S. What do you really think? Patient, clinician and stakeholder views on transdiagnostic mental health treatment for veterans. In: Ametaj A, editor. Transdiagnostic and common element interventions: addressing multidimensional barriers to dissemination and implementation of evidence-based practices. Chicago: Association for Behavioral and Cognitive Therapies; 2015.

55. Creed TA, Stirman SW, Evans AC, Beck AT. A model for implementation of cognitive therapy in community mental health: the Beck initiative. Behav Therapist. 2014;37(3):56-64.

56. Gutner C, Barlow D, Sloan D, Stirman SW, Ametaj A, Chicago IL. What do you really think? Patient, clinician and stakeholder views on transdiagnostic mental health treatment for veterans. In: Association for Behavioral and Cognitive Therapies. Chicago, IL: SRC - GoogleScholar; 2015.

57. Kroenke K, Spitzer RL. The PHQ-9: a new depression diagnostic and severity measure. Psychiatr Ann. 2002;32(9):509-15.

58. Beck AT, Epstein N, Brown GK, Steer RA. An inventory for measuring clinical anxiety: psychometric properties. J Consult Clin Psychol. 1988;56(6):893-7.

59. Rodriguez P, Holowka DW, Marx BP. Assessment of posttraumatic stress disorder-related functional impairment: a review. J Rehabil Res Dev. 2012; 49(5):649-65.

60. Shaw BF, Elkin I, Yamaguchi J, Olmsted M, Vallis TM, Dobson KS, Lowery A, Sotsky SM, Watkins JT, Imber SD. Therapist competence ratings in relation to clinical outcome in cognitive therapy of depression. J Consult Clin Psychol. 1999;67(6):837-46.

61. Dobson KS, Shaw BF, Vallis TM. Reliability of a measure of the quality of cognitive therapy. Br J Clin Psychol. 1985;24(4):295.

62. Beidas R, Marcus S, Aarons GA, Hoagwood KE, Schoenwald SK, Evans AC, Hurford MO, Hadley T, Barg FK, Walsh LM, et al. Predictors of community therapists' use of therapy techniques in a large public mental health system. JAMA Pediatr. 2015.

63. Cook JM, Thompson R, Schnurr PP. Perceived characteristics of intervention scale: development and psychometric properties. Assessment. 2015;22(6):704-14.

64. Aarons GA, Glisson C, Hoagwood K, Kelleher K, Landsverk J, Cafri G. Health TRNoYM: psychometric properties and U.S. national norms of the evidencebased practice attitude scale (EBPAS). Psychol Assess. 2010;22(2):356-65.

65. Carpenter KM, Cheng WY, Smith JL, Brooks AC, Amrhein PC, Wain RM, Nunes EV. "Old dogs" and new skills: how clinician characteristics relate to motivational interviewing skills before, during, and after training. J Consult Clin Psychol. 2012;80(4):560.

66. Damschroder LJ, Aron DC, Keith RE, Kirsh SR, Alexander JA, Lowery JC. Fostering implementation of health services research findings into practice: a consolidated framework for advancing implementation science. Implement Sci. 2009;4(1):50.

67. Shrout PE, Fleiss JL. Intraclass correlations: uses in assessing rater reliability. Psychol Bull. 1979;86(2):420-8.

68. Strunk DR, Brotman MA, DeRubeis RJ, Hollon SD. Therapist competence in cognitive therapy for depression: predicting subsequent symptom change. J Consult Clin Psychol. 2010;78(3):429-37.

69. Cichetti D. Guidelines, criteria, and rules of thumb for normed and standardized instruments in psychology. Psychol Assess. 1994;6(4):284-90.

70. Resick PA, Nishith P, Weaver TL, Astin MC, Feuer CA. A comparison of cognitive-processing therapy with prolonged exposure and a waiting condition for the treatment of chronic posttraumatic stress disorder in female rape victims. J Consult Clin Psychol. 2002;70(4):867.

71. Resick PA, Galovski TE, Uhlmansiek MOB, Scher CD, Clum GA, Young-Xu Y. A randomized clinical trial to dismantle components of cognitive processing therapy for posttraumatic stress disorder in female victims of interpersonal violence. J Consult Clin Psychol. 2008;76(2):243.

72. Zar JH. Spearman rank correlation. In: Armitage P, Colton T, editors. Encyclopedia of Biostat. Chichester: Wiley; 1998.

73. Hastie T, Tibshirani R, Friedman J, Hastie T, Tibshirani R, Friedman J. Crossvalidation. (241-249) New York. NY: Springer New York Inc; 2009.

74. Strunk DR, Brotman MA, DeRubeis RJ. The process of change in cognitive therapy for depression: predictors of early inter-session symptom gains. Behav Res Ther. 2010;48(7):599-606.

75. Barber JP, Triffleman E, Marmar C. Considerations in treatment integrity: implications and recommendations for PTSD research. J Trauma Stress. 2007;20(5):793-805.

76. Hedeker D, Gibbons RD. Application of random-effects pattern-mixture models for missing data in longitudinal studies. Psychol Methods. 1997;2(1):64-78.

77. Altman DG, Schulz KF, Moher D, Egger M, Davidoff F, Elbourne D, Gøtzsche PC, Lang T. The revised CONSORT statement for reporting randomized trials: explanation and elaboration. Ann Intern Med. 2001;134(8):663-94. 
78. Zalta AK, Gillihan SJ, Fisher AJ, Mintz J, McLean CP, Yehuda R, Foa EB. Change in negative cognitions associated with PTSD predicts symptom reduction in prolonged exposure. J Consult Clin Psychol. 2014;82(1):171.

79. Granger CW. Investigating causal relations by econometric models and cross-spectral methods. Econometrica: J Econometric Soc. 1969:424-38.

80. Baldwin SA, Imel ZE, Braithwaite SR, Atkins DC. Analyzing multiple outcomes in clinical research using multivariate multilevel models. J Consult Clin Psychol. 2014;82(5):920.

81. Raudenbush SW. Statistical analysis and optimal design for cluster randomized trials. Psychol Methods. 1997;2(2):173.

82. Snijders TA. Power and sample size in multilevel linear models. Encyclopedia Stat Behav Sci. 2005;3:1570-3.

83. Strunk DR, Cooper AA, Ryan ET, DeRubeis RJ, Hollon SD. The process of change in cognitive therapy for depression when combined with antidepressant medication: predictors of early intersession symptom gains. J Consult Clin Psychol. 2012;80(5):730-8.

84. Committe on Comparative Effectiveness Research Priorization loM. Initial national priotities for comparative effectiveness research. Washington, DC: The National Academies Press; 2009.

85. Glasgow RE, Lichtenstein E, Marcus AC. Why don't we see more translation of health promotion research to practice? Rethinking the efficacy-toeffectiveness transition. Am J Public Health. 2003:93:1261-7.

Ready to submit your research? Choose BMC and benefit from:

- fast, convenient online submission

- thorough peer review by experienced researchers in your field

- rapid publication on acceptance

- support for research data, including large and complex data types

- gold Open Access which fosters wider collaboration and increased citations

- maximum visibility for your research: over $100 \mathrm{M}$ website views per year

At BMC, research is always in progress.

Learn more biomedcentral.com/submissions 